\title{
Newly developed gaseous photomultiplier
}

Fuyuki Tokanai ${ }^{\mathrm{a}}$, Toru Moriya ${ }^{\mathrm{a}}$, Mirei Takeyama ${ }^{\mathrm{a}}$, Hirohisa Sakurai ${ }^{\mathrm{a}}$, Shuichi Gunji ${ }^{\mathrm{a}}$, Takayuki Sumiyoshi ${ }^{\mathrm{b}}$, Takayuki Ito $^{\mathrm{b}}$, Hirioyuki Sugiyamac ${ }^{c}$, Teruyuki Okada ${ }^{c}$, Noboru Ohishic ${ }^{c}$, Syunji Kishimoto ${ }^{d}$

\author{
${ }^{a}$ Department of Physics, Yamagata University, Yamagata 990-8560, Japan \\ ${ }^{b}$ Graduate School of Faculty of Science and Engineering, Tokyo Metropolitan University, Tokyo 192-0397, Japan \\ ${ }^{c}$ Electron Tube Division, Hamamatsu Photonics K.K., Shizuoka 438-0193, Japan \\ ${ }^{d}$ Institute of Material Structure Science, KEK, Tsukuba 305-0801, Japan
}

\begin{abstract}
A new micromesh gas (Micromegas) detector has been developed for a gaseous photomultiplier tube (PMT) with a bialkali photocathode. A basic performance test of the Micromegas detector was carried out for a $\mathrm{Ne}(90 \%)+\mathrm{CF}_{4}(10 \%)$ gas mixture using an X-ray beam. We constructed gaseous PMTs with a bialkali photocathode and Micromegas detectors. The photoelectron collection efficiencies in several gases and the suppression of ion feedback were investigated.
\end{abstract}

Keywords: Gaseous PMT, micropattern gas detector

\section{Introduction}

A potential advantage of the gaseous photomultiplier tube ${ }_{27}$ (PMT) is that it can achieve a very large effective area with ${ }_{28}$ adequate position and timing resolutions. In addition, it can ${ }_{29}^{28}$ be easily operated under a very high magnetic field compared ${ }_{30}$ with a conventional vacuum-based PMT. In the VUV range, $a_{31}$ ring imaging Cherenkov detector (RICH), which consists of $a_{32}$ cesium iodide (CsI) photocathode coupled to a multiwire proportional chamber (MWPC), has been successfully and widely utilized in the field of high-energy physics for particle identification [1]. However, it has been suggested that the effect of ion ${ }_{36}$ feedback causes the aging of the photocathode [1]. In fact, we ${ }_{37}$ observed a degradation of $16 \%$ in the quantum efficiency $(\mathrm{QE})_{38}$ of a gaseous PMT with a CsI photocathode when the accumulated charge due to ion feedback reached $25 \mu \mathrm{C} / \mathrm{mm}^{2}$ [2]. Moreover, we found that this effect was more serious for a gaseous PMT with a bialkali photocathode; the QE was degraded by approximately $20 \%$ when the accumulated charge on the photocathode reached $0.4 \mu \mathrm{C} / \mathrm{mm}^{2}$ [3]. This was due to the work 42 function of the bialkali photocathode being lower than that of the CsI photocathode. The suppression of ion feedback is im- 43 portant to satisfy the demand for higher-sensitivity and long- ${ }_{44}$ term photon detectors.

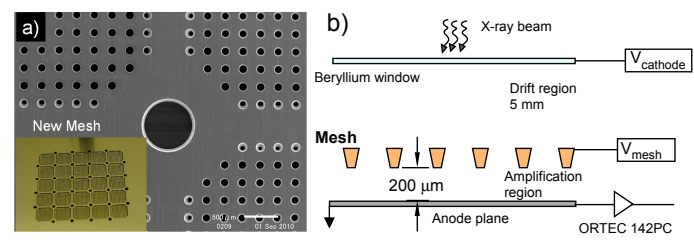

Figure 1: (a) Scanning electron microscopy image of micromesh and (b) ${ }^{53}$ schematic view of new Micromegas detector used for X-ray beam testing.

The micromesh gas (Micromegas [4]) detector is a very at- 56 tractive MPGD for application to the gaseous PMT because it can reach a sufficient gain of up to $10^{5}$ and the rate of ion backflow decreases to $10^{-3}$ when it is used a stand-alone detector without requiring an additional MPGD [5]. To satisfy the conditions required for gaseous PMT production, we have developed a new Micromegas detector with the same metal material and insulator as those used in vacuum-type PMTs. Firstly, we investigated the basic performance of the new Micromegas detector with an X-ray beam. Following the success of the X-ray beam test, we developed a gaseous PMT consisting of a bialkali photocathode and Micromegas detectors. Arranging two types of micromeshes in a staggered pattern in a double Micromegas detector, we successfully reduced the ion feedback to $7 \times 10^{-4}$ in a gaseous PMT. In this paper, we describe the basic performance of the gaseous PMT, the photoelectron collection efficiency, and our attempt to suppress ion feedback of the gaseous PMT.

\section{Newly Developed Gaseous PMT}

\subsection{New Micromesh Gas Detector}

Fig. 1 a) shows the micromesh used in the Micromegas detector. The new mesh is fabricated by chemical etching of a single alloy plate. The chemical etching produces a single conical hole on the metal of $46 \mu \mathrm{m}$ thickness. The diameters of the small and large holes of the mesh are $80 \mu \mathrm{m}$ and $120 \mu \mathrm{m}$, respectively. The pitch of each hole is $250 \mu \mathrm{m}$. The gas gain and energy resolution of the Micromegas detector were investigated for a filling gas of $\mathrm{Ne}(90 \%)+\mathrm{CF}_{4}(10 \%)$ gas mixture at 760 torr using the X-ray beams at the BL14A beamline in KEK-PF. The typical setup of the Micromegas detector for Xray detection is shown in Fig. 1 b). The drift and absorption region for X-rays is $5 \mathrm{~mm}$ between the beryllium window and the top of the mesh. The amplification gap is normally in the 
range from 100 to $200 \mu \mathrm{m}$, which is defined by the ceramic ${ }_{84}$ spacers set on the anode plate at regular intervals of $6.4 \mathrm{~mm} .85$ The energies of the incident $\mathrm{X}$-ray beams were fixed at $6 \mathrm{keV}{ }_{86}$ by a Si double-crystal monochromator. The incident X-rays ${ }_{87}$ were defined by a collimator of $0.1 \mathrm{~mm}$ diameter. The electric 88 field in the drift region was set at $100 \mathrm{~V} / \mathrm{cm}$. The charge signals 89 from the anode plate were measured to identify the energy de- 90 posited by each incident X-ray event. Fig. 2 a) shows the gain 91 as a function of the voltage between the mesh and the anode 92 plate. Gains of up to $2 \times 10^{4}$ were safely achieved using a sin- 93 gle mesh. The typical pulse height spectrum for $6 \mathrm{keV}$ X-rays 94 is shown in Fig. 2 b) at a gas gain of 3000. The energy resolu- 95 tion was $18 \%$ (FWHM), which is consistent with that of $17 \% 96$ obtained using a hole-type micropattern gas detector (MPGD) 97 with a glass capillary plate [6].
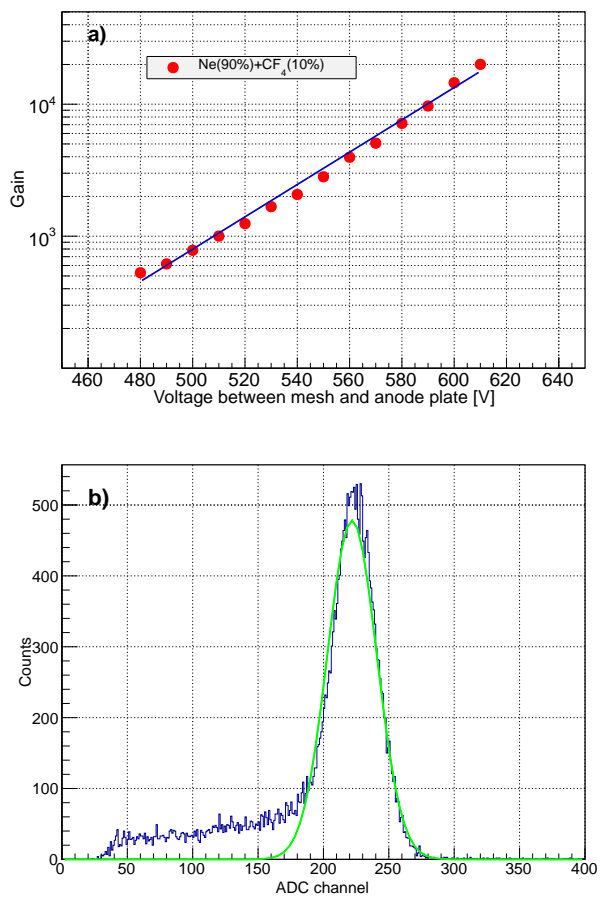

Figure 2: (a) Gain as a function of voltage between mesh and anode plate of Micromegas detector. (b) Pulse height distribution of charge signal of the Micromegas detector for X-ray beams. The energy resolution was 18\% (FWHM) for $6 \mathrm{keV} \mathrm{X}$-rays.

\subsection{Photoelectron Collection Efficiency in Gases}

The sensitivity of a PMT is described in terms of its QE. ${ }_{116}$ The $\mathrm{QE}$ is very important in many experiments, particularly ${ }_{117}$ when dealing with low photon statistics. The QE is defined ${ }_{118}$ as the ratio of the number of photoelectrons emitted from ${ }_{119}$ the photocathode to that of incident photons. In a previous 120 work [7], we found that the maximum QEs of gaseous PMTs 121 filled with $\mathrm{Ar}(90 \%)+\mathrm{CH}_{4}(10 \%)$ and $\left.\mathrm{Ne}(90 \%)+\mathrm{CF}_{4}(10 \%)\right)_{122}$ were $14 \%$ and $12 \%$, respectively, whereas the average $\mathrm{QE}$ of ${ }_{123}$ the same PMT in a vacuum environment was approximately ${ }_{124}$ $20 \%$ at wavelengths between 300 and $500 \mathrm{~nm}$ before gas fill-125 ing. The difference in $\mathrm{QE}$ after filling the gas can be explained ${ }_{126}$ by the effect of photoelectron backscattering due to collisions with the gas molecules. We investigate the photoelectron collection efficiency from the bialkali photocathode of a gaseous PMT filled with various gas mixtures of $\mathrm{Ar}(90 \%)+\mathrm{CH}_{4}(10 \%)$, $\mathrm{Ne}(90 \%)+\mathrm{CF}_{4}(10 \%), \mathrm{Ne}(90 \%)+\mathrm{i}-\mathrm{C}_{4} \mathrm{H}_{10}(10 \%)$ and pure $\mathrm{Xe}$ to evaluate the effect of photoelectron backscattering. Fig. 3 shows the experimental setup. The gaseous PMT consists of a bialkali photocathode, the micromesh described above and an anode plate. The photocathode was irradiated with visible light and the photocurrent was measured from the mesh electrode. The photoelectron collection efficiency is defined as the ratio of the photocurrent in the gas to that obtained with the same PMT operating in vacuum. Fig. 4 shows the photoelectron collection efficiency for each filling gas as a function of electric field at a gas pressure of 675 torr. The efficiency for each gas increases rapidly up to an electric field of $50 \mathrm{~V} / \mathrm{cm}$, then increases gradually. The highest and lowest photoelectron collection efficiencies were obtained for $\mathrm{Ar}(90 \%)+\mathrm{CH}_{4}(10 \%)$ and $\mathrm{Xe}(100 \%)$, respectively. The efficiency for $\mathrm{Ne}(90 \%)+\mathrm{CF}_{4}$ $(10 \%)$ is 1.6 times higher than that for $\mathrm{Ne}(90 \%)+\mathrm{i}-\mathrm{C}_{4} \mathrm{H}_{10}(10 \%)$ at an electric field of $\sim 150 \mathrm{~V} / \mathrm{cm}$. The dependence of the photoelectron collection efficiency on the gas pressure is shown in Fig. 5 for the gas mixture of $\mathrm{Ne}(90 \%)+\mathrm{CF}_{4}(10 \%)$. This figure indicates that the dependence of the photoelectron collection efficiency on the applied electric field is approximately the same at each pressure, and that the photoelectron collection efficiency increases with decreasing pressure. A sudden increase in the efficiency is observed at an electric field of $\sim 250 \mathrm{~V} / \mathrm{cm}$ for the gas pressure of 75 torr. It is assumed that photon feedback produced by the onset of gas excitation occurs at lower gas pressures.
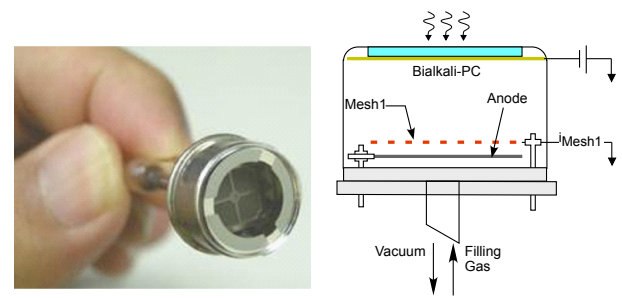

Figure 3: Experimental setup for measurement of the photoelectron collection efficiency with a gaseous PMT consisting of a bialkali photocathode and a Micromegas detecter.

\subsection{Attempt to Suppress Ion Feedback}

To suppress ion backflow from the electron multiplication stage in a gaseous PMT, we have adopted the new idea of arranging two micromeshes as a staggered pattern in a double Micromegas detector [8,9]. Fig. 6 shows a schematic view of the gaseous PMT using the double Micromegas detector with a bialkali photocathode. Micromeshes with different hole diameters of $100 \mu \mathrm{m}$ (upper) and $190 \mu \mathrm{m}$ (lower) are arranged in a staggered pattern. A simulated single electron avalanche is also shown in Fig. 6 for a $\mathrm{Ne}(90 \%)+\mathrm{CF}_{4}(10 \%)$ gas mixture that was obtained using the Maxwell 3D [10] and GARFIELD codes [11]. It is clear that almost all the ions produced in the 


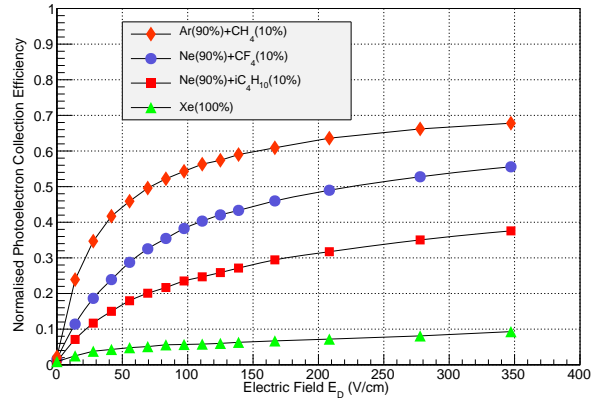

Figure 4: Photoelectron collection efficiencies for a gaseous PMT filled with various gases as a function of electric field.

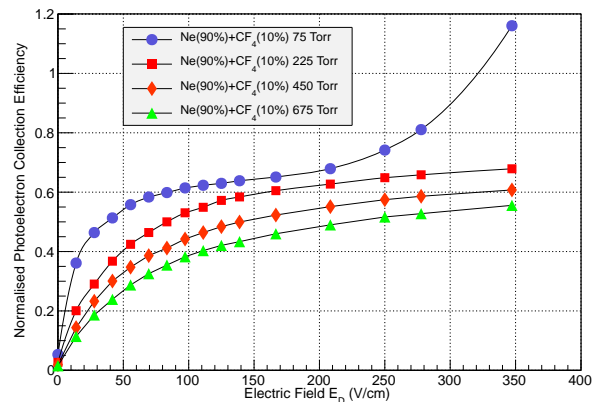

Figure 5: Photoelectron collection efficiencies for a gaseous PMT filled with ${ }^{151}$ different pressures of $\mathrm{Ne}(90 \%)+\mathrm{CF}_{4}(10 \%)$ as a function of electric field. 152 multiplication region are collected by the first micromesh and ${ }^{154}$ that few ions reach the drift region.

The characteristics of the gaseous PMT with the double Mi- ${ }^{156}$ cromegas detector having two staggered micromeshes and $\mathrm{a}^{157}$ bialkali photocathode were investigated for a $\mathrm{Ne}(90 \%)+\mathrm{CF}_{4}{ }_{45}$ $(10 \%)$ gas mixture at 675 torr. The detailed experimental setup ${ }^{159}$ is described in [12]. The ion feedback (IBF) of the gaseous PMT was defined as

$$
I B F=\frac{I_{\text {Cathode }}-I_{0}}{I_{\text {Anode }}},
$$

where $I_{\text {Cathode }}$ and $I_{\text {Anode }}$ are the electrical currents on the photocathode and the anode, respectively, and $I_{0}$ is the primary cur-164 rent on the photocathode. The gain of the gaseous PMT was de-165 rived from the ratio of the anode current $\left(I_{\text {Anode }}\right)$ to the primary 166 current $\left(I_{0}\right):$ Gain $=I_{\text {Anode }} / I_{0}$. Fig. 7 shows the effective gain and ion feedback as functions of voltage applied between the second mesh and the anode electrode. We obtained a maximum gain of up to $10^{4}$ and ion feedback of less than $6 \times 10^{-4} \cdot{ }_{168}$

\section{Conclusion}

We have been developing a gaseous PMT with a bialkali pho- ${ }^{17}$ tocathode and MPGDs. A new Micromegas detector was devel- ${ }^{174}$ oped using a suitable material for the production of a bialkali ${ }_{176}^{175}$ photocathode in a gaseous PMT. A performance test was car-177 ried out using an X-ray beam. The Micromegas detector was ${ }^{178}$ operated in a $\mathrm{Ne}(90 \%)+\mathrm{CF}_{4}(10 \%)$ gas mixture at 760 torr. $^{179}$
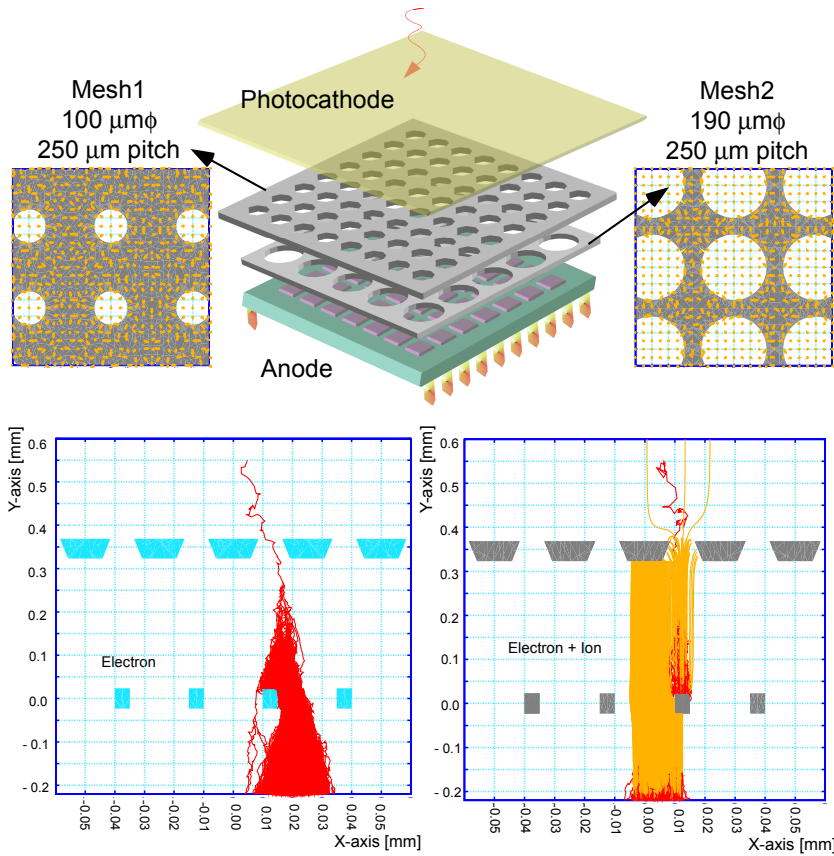

Figure 6: Schematic view of gaseous PMT with a bialkali photocathode and double Micromegas detector. A single electron avalanche in the double Micromegas detector was simulated.

We successfully obtained a gain of $2 \times 10^{4}$ and an energy resolution of $18 \%$ for $6 \mathrm{keV}$ X-rays. A sealed gaseous PMT consisting of a bialkali photocathode and the Micromegas detector was fabricated. We investigated the photoelectron collection efficiency of the gaseous PMT in several gases. The highest photoelectron collection efficiency was obtained for $\mathrm{Ar}(90 \%)+\mathrm{CH}_{4}$ (10\%) among these gases. We developed a gaseous PMT by arranging two micromeshes in a staggered pattern, which was then tested. A maximum gain of up to $10^{4}$ and ion feedback of less than $6 \times 10^{-4}$ were obtained. We are currently developing a flat-type gaseous PMT with bialkali photocathode using two micromeshes in a staggered pattern for practical use.

\section{Acknowledgements}

This work was supported by a JSPS Grant-in-Aid and the JST Advanced Characterization Technology and Instrument Development Project.

\section{References}

[1] H. Hoedlmoser, et al.,, Nucl. Instr. and Meth. A 574 (2007) 28.

[2] F. Tokanai, et al., Nucl. Instr. and Meth. A 628 (2011) 190

[3] T. Moriya, et al., Nucl. Instr. and Meth. A 732 (2013) 269.

[4] Y. Giomataris, Nucl. Instr. and Meth. A 376 (1996) 84

[5] P. Colas et al., Nucl. Instr. and Meth. A 535 (2004) 226

[6] F. Tokanai, et al., Nucl. Instr. and Meth. A 581 (2007) 236

[7] F. Tokanai, et al., Nucl. Instr. and Meth. A 610 (2009) 164

[8] F. Jeanneau, et al., Nucl. Instr. and Meth. A 623 (2010) 94.

[9] K. Matsumoto, et al., Phys. Proc. 37 (2012) 499.

[10] MAXWELL 3D Field Simulation, Userfs reference, Ansoft Corporation.

[11] GARFIELD, CERN Simulation of gaseous detector, R. Vennhof.

[12] T. Ito, et al., IEEE NSS-MIC 2013 Conference Record. 

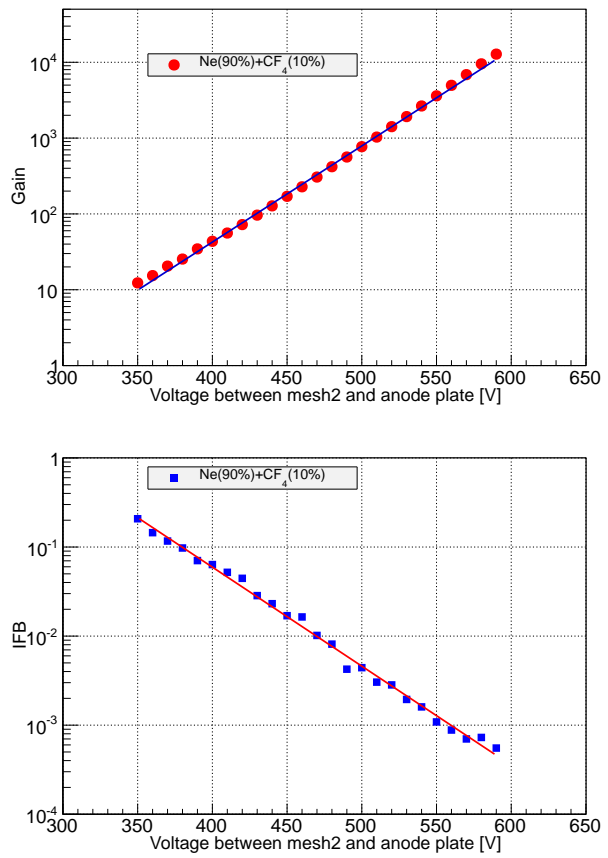

Figure 7: Gain and ion feedback of the gaseous PMT with a bialkali photocathode and doulbe Micromegas detector. 